\title{
Asymptotic iteration method for solving Hahn difference equations
}

\author{
Lucas MacQuarrie ${ }^{1}$, Nasser Saad ${ }^{1 *}$ (D) and Md. Shafiqul Islam¹
}

\author{
*Correspondence: nsaad@upei.ca \\ 'school of Mathematical and \\ Computational Sciences, University \\ of Prince Edward Island, \\ Charlottetown, Canada
}

\begin{abstract}
Hahn's difference operator $D_{q ; w} f(x)=(f(q x+w)-f(x)) /((q-1) x+w), q \in(0,1), w>0$, $x \neq w /(1-q)$ is used to unify the recently established difference and $q$-asymptotic iteration methods (DAIM, qAIM). The technique is applied to solve the second-order linear Hahn difference equations. The necessary and sufficient conditions for polynomial solutions are derived and examined for the $(q ; w)$-hypergeometric equation.
\end{abstract}

MSC: 39A05; 39A06; 39A13; 39A60; 47B39

Keywords: Hahn operator; Linear difference equations; q-difference equations; Polynomial solutions; Eigenvalue problems

\section{Introduction}

Hahn $[1,2]$ introduced, for $x \neq w /(1-q)$, the quantum difference operator

$$
\begin{aligned}
& D_{q ; w} f(x)=\frac{f(q x+w)-f(x)}{(q-1) x+w}, \quad q \in(0,1), w>0, \\
& D_{q ; w}^{0} f(x)=f(x), \quad D_{q ; w}^{n} f(x)=D_{q ; w}\left\{D_{q ; w}^{n-1} f(x)\right\}, \quad n=1,2, \ldots,
\end{aligned}
$$

where $f$ is a function defined on an interval $I$ of $\mathbb{R}$ which contains $w /(1-q)$. It is clear, using L'Hopital's rule, that

$$
\lim _{x \rightarrow w /(1-q)} D_{q ; w} f(x)=f^{\prime}\left(\frac{w}{1-q}\right)
$$

provided that the function $f$ is differentiable at $w /(1-q)$ in the usual sense.

The Hahn difference operator $D_{q ; w}$ unifies (in the limit) and generalizes two well-known difference operators; namely, the quantum $q$-difference operator $D_{q} \equiv D_{q ; 0}$ (see [3-7]) and the forward difference operator $\Delta_{w} \equiv D_{1 ; w}$ (see $\left.[8,9]\right)$. Included as a special case is the discrete forward difference operator $\Delta=D_{1 ; 1}$. In the limit, both $D_{q}$ and $\Delta_{w}$ generalize the derivative operator $f^{\prime}(x)=d f / d x$. The generalization and the limit process to these

(c) The Author(s) 2021. This article is licensed under a Creative Commons Attribution 4.0 International License, which permits use, sharing, adaptation, distribution and reproduction in any medium or format, as long as you give appropriate credit to the original author(s) and the source, provide a link to the Creative Commons licence, and indicate if changes were made. The images or other third party material in this article are included in the article's Creative Commons licence, unless indicated otherwise in a credit line to the material. If material is not included in the article's Creative Commons licence and your intended use is not permitted by statutory regulation or exceeds the permitted use, you will need to obtain permission directly from the copyright holder. To view a copy of this licence, visit http://creativecommons.org/licenses/by/4.0/. 
important operators is illustrated by the following diagram:

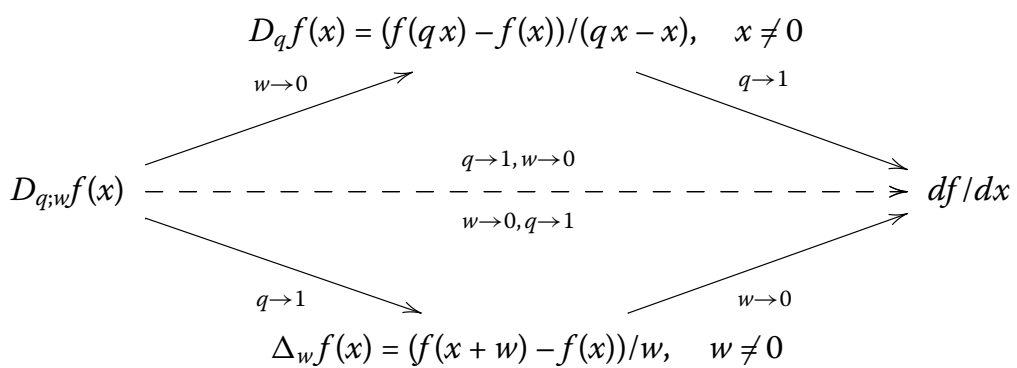

A rigorous analysis of the calculus associated with the operator $D_{q ; w}$ along with the construction of a proper inverse of $D_{q ; w}$ and the associated integral calculus can be found in [10-12].

The Hahn operator $D_{q ; w}$ is an important tool in the construction of families of orthogonal polynomials and several approximation problems (see, for example, [13-19]).

In 2003, Çiftçi, Hall, and Saad introduced [20, 21] the asymptotic iteration method (AIM) to solve analytically and/or approximately the second-order linear homogeneous differential equation

$$
y^{\prime \prime}(x)=\lambda_{0}(x) y^{\prime}(x)+s_{0}(x) y(x), \quad{ }^{\prime}=d / d x,
$$

where $\lambda_{0}(x)$ and $s_{0}(x)$ are continuously differentiable functions. They proved that, up to some multiplication constant, an analytic solution of this differential equation reads

$$
y(x)=\exp \left(-\int^{x} \frac{s_{n-1}(t)}{\lambda_{n-1}(t)} d t\right)
$$

provided that, for some $n>0$, (so-called terminating condition)

$$
\frac{s_{n}(x)}{\lambda_{n}(x)}=\frac{s_{n-1}(x)}{\lambda_{n-1}(x)} \quad \text { or } \quad \delta_{n}(x)=\lambda_{n}(x) s_{n-1}(x)-s_{n}(x) \lambda_{n-1}(x) \equiv 0
$$

given the AIM-sequences, for $n=0,1,2, \ldots$, as

$$
\begin{aligned}
& \lambda_{n}(x)=\lambda_{n-1}^{\prime}(x)+\lambda_{n-1}(x) \lambda_{0}(x)+s_{n-1}(x), \\
& s_{n}(x)=s_{n-1}^{\prime}(x)+s_{0}(x) \lambda_{n-1}(x),
\end{aligned}
$$

initiated with $\lambda_{-1}=1$ and $s_{-1}=0$. This powerful and simple technique [22] proved to be very useful in studying the eigenvalue problems in quantum mechanics. Noting that if the analytic solutions of the linear differential equation (2) are not available, the terminating condition (4) plays a vital role in approximating the exact solutions with high (and almost controllable) precision [23, 24]. Ismail and Saad [25] recently shed further insight on the AIM mathematical foundation where the reasons for its success and the possible convergence failures of the iterative aspect of it were presented. 
In another study by Ismail and Saad [26], a discrete version of the AIM was presented, $d A I M$, along with applications to the classical difference equations [27]. Given the secondorder linear difference equation

$$
\Delta^{2} y(x)=\lambda_{0}(x) \Delta y(x)+s_{0}(x) y(x), \quad x \geq x_{0},
$$

a solution, up to some constant (periodic) function, is given by

$$
y(x)=\prod_{i=x_{0}}^{x-1}\left(1-\frac{s_{n-1}(i)}{\lambda_{n-1}(i)}\right)
$$

provided that, for some $n>0$,

$$
\frac{s_{n}(x)}{\lambda_{n}(x)}=\frac{s_{n-1}(x)}{\lambda_{n-1}(x)}, \quad n=1,2, \ldots,
$$

where

$$
\begin{aligned}
& \lambda_{n}(x)=\Delta \lambda_{n-1}(x)+\lambda_{n-1}(x+1) \lambda_{0}(x)+s_{n-1}(x+1), \\
& s_{n}(x)=\Delta s_{n-1}(x)+\lambda_{n-1}(x+1) s_{0}(x) .
\end{aligned}
$$

Also, the quantum $q$-discrete version $q A I M$ was introduced in [26]. Given a second-order $q$-difference equation

$$
D_{q}^{2} y(x)=\lambda_{0}(x) D_{q} y(x)+s_{0}(x) y(x), \quad 0<q<1,
$$

a solution, up to some constant periodic function, is given by

$$
y(x)=\prod_{k=0}^{\infty}\left[1+(1-q) q^{k} x \frac{s_{n-1}\left(q^{k} x\right)}{\lambda_{n-1}\left(q^{k} x\right)}\right]^{-1}
$$

provided that

$$
\frac{s_{n}(x)}{\lambda_{n}(x)}=\frac{s_{n-1}(x)}{\lambda_{n-1}(x)}, \quad n=1,2, \ldots,
$$

where the functions $\lambda_{n}(x)$ and $s_{n}(x)$ are generated by

$$
\begin{aligned}
& \lambda_{n}(x)=D_{q} \lambda_{n-1}(x)+\lambda_{n-1}(q x) \lambda_{0}(x)+s_{n-1}(q x), \\
& s_{n}(x)=D_{q} s_{n-1}(x)+\lambda_{n-1}(q x) s_{0}(x) .
\end{aligned}
$$

For the proofs and detailed examples, we refer the reader to the recently published manuscript [26].

In the present work, we unify both the $d$ AIM and $q$ AIM using the Hahn operator (1). To achieve this goal, in Sect. 2 we summarize the relevant definitions and properties of the Hahn difference operator. Several novel properties of this operator are also introduced and proved in that section. In particular, the polynomials in the $(q ; w)$-space are introduced, 
and the associated Taylor polynomial expansions are derived. In Sect. 3, the solutions of the first- and second-order linear Hahn difference equations

$$
\left\{\begin{array}{l}
D_{q ; w} y(x)=\alpha(x) y(x)+\beta(x), \\
D_{q: w}^{2} y(x)=\lambda_{0}(x) D_{q: w} y(x)+s_{0}(x) y(x),
\end{array}\right.
$$

are examined. The necessary and sufficient conditions for the existence of polynomial solutions of the second-order linear Hahn difference equation are derived and proved. In Sect. 4, the solutions of the hypergeometric equation [28]

$$
\left(e x^{2}+2 f x+g\right) D_{q ; w}^{2} y(x)+(2 \varepsilon x+\gamma) D_{q ; w} y(x)+\tau y(x)=0
$$

are deduced. The necessary and sufficient conditions of the polynomial solutions are established and applied to construct the first few solutions explicitly.

\section{Preliminary definitions and properties of Hahn operator}

To make this paper self-contained, we review the mathematical properties of the Hahn operator $[1,2,10,11,18,29-32]$ in this section and provide the proofs for other new properties developed for the present work.

Theorem 1 Iff, $g: I \rightarrow \mathbb{R}$ and $a, b \in \mathbb{R}$ are $q ; w$-differentiable functions and $x \in I$, then:

1. Linearity:

$$
D_{q ; w}[a f(x)+b g(x)]=a D_{q ; w} f(x)+b D_{q ; w} g(x) .
$$

2. Product rule:

$$
\begin{aligned}
D_{q ; w}(f(x) g(x)) & =f(q x+w) D_{q ; w} g(x)+g(x) D_{q ; w} f(x) \\
& =g(q x+w) D_{q ; w} f(x)+f(x) D_{q ; w} g(x) .
\end{aligned}
$$

3. Quotient rule:

$$
\begin{aligned}
D_{q ; w}\left(\frac{f(x)}{g(x)}\right) & =\frac{g(x) D_{q ; w} f(x)-f(x) D_{q ; w} g(x)}{g(q x+w) g(x)} \\
& =\frac{g(q x+w) D_{q ; w} f(x)-f(q x+w) D_{q ; w} g(x)}{g(q x+w) g(x)},
\end{aligned}
$$

provided that $g(q x+w) g(x) \neq 0$ for all $x \in I$.

Lemma 1 For $q \neq 0$ and $n=1,2, \ldots$,

$$
\begin{aligned}
f\left(q^{n} x+w \sum_{j=0}^{n-1} q^{j}\right)= & f(x) \\
& +((q-1) x+w) \sum_{j=0}^{n-1} q^{j} D_{q ; w} f\left(q^{j} x+w \sum_{i=0}^{j-1} q^{i}\right) .
\end{aligned}
$$


Proof By definition

$$
[(q-1) x+w] D_{q ; w} f(x)=f(q x+w)-f(x)
$$

thus, replacing $x$ with $q x+w$, it follows that

$$
q[(q-1) x+w] D_{q ; w} f(q x+w)=f\left(q^{2} x+\sum_{j=0}^{1} q^{j} w\right)-f(q x+w) .
$$

Repeating the process, we have recursively that

$$
\begin{aligned}
q^{k} & {[(q-1) x+w] D_{q ; w} f\left(q^{k} x+\sum_{j=0}^{k-1} q^{j} w\right) } \\
& =f\left(q^{k+1} x+\sum_{j=0}^{k} q^{j} w\right)-f\left(q^{k} x+\sum_{j=0}^{k-1} q^{j} w\right) .
\end{aligned}
$$

The assertion then follows by sum of these equations for $j=0$ to $k$.

The following gives a generalization to Lemma 1.

Lemma 2 For $x \neq w /(1-q)$,

$$
\begin{aligned}
\sum_{j=0}^{n} q^{j} f\left(q^{j} x+w \sum_{i=0}^{j-1} q^{i}\right) D_{q ; w} g\left(q^{j} x+w \sum_{i=0}^{j-1} q^{i}\right) \\
=\frac{f\left(q^{n+1} x+w \sum_{j=0}^{n} q^{j}\right) g\left(q^{n+1} x+w \sum_{j=0}^{n} q^{j}\right)-f(x) g(x)}{(q-1) x+w} \\
\quad-\sum_{j=0}^{n} q^{j} g\left(q^{j}(q x+w)+w \sum_{i=0}^{j-1} q^{i}\right) D_{q ; w} f\left(q^{j} x+w \sum_{i=0}^{j-1} q^{i}\right),
\end{aligned}
$$

where identity (19) follows for $g(x)$ or $f(x)$ is a constant function.

Proof We note

$$
\begin{aligned}
& \sum_{j=0}^{n} q^{j} D_{q ; w}\left[f\left(q^{j} x+w \sum_{i=0}^{j-1} q^{i}\right) g\left(q^{j} x+w \sum_{i=0}^{j-1} q^{i}\right)\right] \\
& =\frac{f\left(q^{n+1} x+w \sum_{j=0}^{n} q^{j}\right) g\left(q^{n+1} x+w \sum_{j=0}^{n} q^{j}\right)-f(x) g(x)}{(q-1) x+w} .
\end{aligned}
$$

By the product rule of $D_{q ; w}\left[f\left(q^{j} x+w \sum_{i=0}^{j-1} q^{i}\right) g\left(q^{j} x+w \sum_{i=0}^{j-1} q^{i}\right)\right]$, see (17), we have

$$
\begin{gathered}
\sum_{j=0}^{n} q^{j}\left[g\left(q^{j}(q x+w)+w \sum_{i=0}^{j-1} q^{i}\right) D_{q ; w} f\left(q^{j} x+w \sum_{i=0}^{j-1} q^{i}\right)\right. \\
\left.+f\left(q^{j} x+w \sum_{i=0}^{j-1} q^{i}\right) D_{q ; w} g\left(q^{j} x+w \sum_{i=0}^{j-1} q^{i}\right)\right]
\end{gathered}
$$




$$
=\frac{f\left(q^{n+1} x+w \sum_{j=0}^{n} q^{j}\right) g\left(q^{n+1} x+w \sum_{j=0}^{n} q^{j}\right)-f(x) g(x)}{(q-1) x+w},
$$

which completes the proof after some simple arrangements.

Theorem 2 For a q; w-differentiable function $f$,

$$
f\left(q^{n} x+w \sum_{k=0}^{n-1} q^{k}\right)=\sum_{k=0}^{n}\left(\begin{array}{l}
n \\
k
\end{array}\right)_{q} q^{\frac{1}{2} k(k-1)}[(q-1) x+w]^{k} D_{q ; w}^{k} f(x),
$$

where $\left(\begin{array}{l}n \\ k\end{array}\right)_{q}$ is the q-binomial coefficients [27, formula 12.1.4].

Proof We prove this by induction on $n$. The result holds for $n=1$ by the definition

$$
f(q x+w)=f(x)+[(q-1) x+w] D_{q ; w} f(x) .
$$

For the inductive step, assume that for some $n$ the theorem is true. For $n+1$, it follows using the well-know identity [33], for $n \geq 1$ and $1 \leq k \leq n$,

$$
\left(\begin{array}{c}
n+1 \\
k
\end{array}\right)_{q}=\left(\begin{array}{l}
n \\
k
\end{array}\right)_{q}+q^{n+1-k}\left(\begin{array}{c}
n \\
k-1
\end{array}\right)_{q}
$$

that

$$
\begin{aligned}
\sum_{k=0}^{n+1}\left(\begin{array}{c}
n+1 \\
k
\end{array}\right)_{q} q^{k(k-1) / 2}[(q-1) x+w]^{k} D_{q ; w}^{k} f(x) \\
=\sum_{k=0}^{n+1}\left(\begin{array}{l}
n \\
k
\end{array}\right) q_{q}^{k(k-1) / 2}[(q-1) x+w]^{k} D_{q ; w}^{k} f(x) \\
\quad+\sum_{k=0}^{n+1} q^{n+1-k}\left(\begin{array}{c}
n \\
k-1
\end{array}\right)_{q} q^{k(k-1) / 2}[(q-1) x+w]^{k} D_{q ; w}^{k} f(x) .
\end{aligned}
$$

However, using the properties of the $q$-binomial coefficient $\left(\begin{array}{c}n \\ n+1\end{array}\right)_{q}=0$ and $\left(\begin{array}{c}n \\ 0\end{array}\right)_{q}=1$, we have

$$
\begin{aligned}
\sum_{k=0}^{n+1}\left(\begin{array}{c}
n+1 \\
k
\end{array}\right)_{q} q^{k(k-1) / 2}[(q-1) x+w]^{k} D_{q ; w}^{k} f(x) \\
=\sum_{k=0}^{n}\left(\begin{array}{l}
n \\
k
\end{array}\right) q_{q}^{k(k-1) / 2}[(q-1) x+w]^{k} D_{q ; w}^{k} f(x) \\
\quad+\sum_{k=1}^{n+1} q^{n+1-k}\left(\begin{array}{c}
n \\
k-1
\end{array}\right)_{q} q^{k(k-1) / 2}[(q-1) x+w]^{k} D_{q ; w}^{k} f(x) .
\end{aligned}
$$

For $1 \leq k \leq n+1$, we have for $j=k-1$, where $0 \leq j \leq n$, that

$$
\begin{aligned}
& \sum_{k=0}^{n+1}\left(\begin{array}{c}
n+1 \\
k
\end{array}\right)_{q} q^{k(k-1) / 2}[(q-1) x+w]^{k} D_{q ; w}^{k} f(x) \\
& \quad=\sum_{k=0}^{n}\left(\begin{array}{l}
n \\
k
\end{array}\right) q_{q} q^{k(k-1) / 2}[(q-1) x+w]^{k} D_{q ; w}^{k} f(x)
\end{aligned}
$$




$$
\begin{aligned}
& +\sum_{j=0}^{n} q^{n-j}\left(\begin{array}{l}
n \\
j
\end{array}\right)_{q} q^{(j+1) j / 2}[(q-1) x+w]^{j+1} D_{q ; w}^{j+1} f(x) \\
= & f\left(q^{n} x+w \sum_{k=0}^{n-1} q^{k}\right)+q^{n}[(q-1) x+w] \\
& \times \sum_{j=0}^{n}\left(\begin{array}{l}
n \\
j
\end{array}\right)_{q} q^{j(j-1) / 2}[(q-1) x+w]^{j} D_{q ; w}^{j}\left(D_{q ; w} f(x)\right),
\end{aligned}
$$

which, using (20), finally implies

$$
\begin{aligned}
& \sum_{k=0}^{n+1}\left(\begin{array}{c}
n+1 \\
k
\end{array}\right) q_{q}^{k(k-1) / 2}[(q-1) x+w]^{k} D_{q ; w}^{k} f(x) \\
& \quad=f\left(q^{n} x+w \sum_{k=0}^{n-1} q^{k}\right)+q^{n}[(q-1) x+w] D_{q ; w} f\left(q^{n} x+w \sum_{k=0}^{n-1} q^{k}\right) \\
& \quad=f\left(q^{n+1} x+w \sum_{k=0}^{n} q^{k}\right) .
\end{aligned}
$$

This completes the proof.

For example, if $n=3$, then this lemma implies

$$
\begin{aligned}
& f\left(q^{3} x+w \sum_{i=0}^{2} q^{i}\right) \\
& =f(x)+\left(1+q+q^{2}\right)((q-1) x+w) D_{q ; w} f(x) \\
& \quad+q\left(q^{2}+q+1\right)((q-1) x+w)^{2} D_{q ; w}^{2} f(x)+q^{3}((q-1) x+w)^{3} D_{q ; w}^{3} f(x) .
\end{aligned}
$$

A slightly different form of the following theorem was given in [34, Lemma 2.1]; however, we give here a direct proof based on the method of mathematical induction.

Theorem 3 Let $f$ be a $q$; $w$ differentiable function on I. Then, for $x \neq w /(1-q)$, the $n$th $q ; w$ derivative is evaluated using

$$
\begin{aligned}
D_{q ; w}^{n} f(x)= & \frac{q^{-n(n-1) / 2}}{((q-1) x+w)^{n}} \\
& \times \sum_{k=0}^{n}(-1)^{n-k} q^{(n-k)(n-k-1) / 2}\left(\begin{array}{l}
n \\
k
\end{array}\right){ }_{q} f\left(q^{k} x+w \sum_{i=0}^{k-1} q^{i}\right) .
\end{aligned}
$$

Proof Again, by induction on $n$, the result holds for $n=1$ by

$$
D_{q ; w} f(x)=((q-1) x+w)^{-1}[f(q x+w)-f(x)] .
$$


Assume it is true for $n$. Then, for $n+1$,

$$
\begin{aligned}
D_{q ; w}^{n+1} f(x) & =D_{q ; w}^{n}\left(D_{q ; w} f(x)\right) \\
& =\frac{q^{-n(n-1) / 2}}{((q-1) x+w)^{n}} \sum_{k=0}^{n}(-1)^{n-k} q^{(n-k)(n-k-1) / 2}\left(\begin{array}{l}
n \\
k
\end{array}\right)_{q} D_{q ; w} f\left(q^{k} x+w \sum_{i=0}^{k-1} q^{i}\right) .
\end{aligned}
$$

However, by definition,

$$
D_{q ; w} f\left(q^{k} x+w \sum_{i=0}^{k-1} q^{i}\right)=\frac{f\left(q^{k+1} x+w \sum_{j=0}^{k} q^{j}\right)-f\left(q^{k} x+w \sum_{j=0}^{k-1} q^{j}\right)}{q^{k}[(q-1) x+w]}
$$

it follows that

$$
\begin{aligned}
D_{q ; w}^{n+1} f(x)= & \frac{q^{-n(n-1) / 2}}{((q-1) x+w)^{n+1}} \\
& \times \sum_{k=0}^{n}(-1)^{n-k} q^{(n-k)(n-k-1) / 2-k}\left(\begin{array}{l}
n \\
k
\end{array}\right) f\left(q_{q}^{k+1} x+w \sum_{i=0}^{k} q^{i}\right) \\
& -\frac{q^{-n(n-1) / 2}}{((q-1) x+w)^{n+1}} \\
& \times \sum_{k=0}^{n}(-1)^{n-k} q^{(n-k)(n-k-1) / 2-k}\left(\begin{array}{l}
n \\
k
\end{array}\right)_{q} f\left(q^{k} x+w \sum_{i=0}^{k-1} q^{i}\right) .
\end{aligned}
$$

Shifting the index in the first sum, it follows that

$$
\begin{aligned}
D_{q ; w}^{n+1} f(x)= & q^{-n(n-1) / 2}((q-1) x+w)^{-n-1} \\
& \times \sum_{k=1}^{n+1}(-1)^{n-k+1} q^{(n-k+1)(n-k) / 2-k+1}\left(\begin{array}{c}
n \\
k-1
\end{array}\right) f\left(q^{k} x+w \sum_{i=0}^{k-1} q^{i}\right) \\
& -q^{-n(n-1) / 2}((q-1) x+w)^{-n-1} \\
& \times \sum_{k=0}^{n}(-1)^{n-k} q^{(n-k)(n-k-1) / 2-k}\left(\begin{array}{l}
n \\
k
\end{array}\right) f\left(q^{k} x+w \sum_{i=0}^{k-1} q^{i}\right)
\end{aligned}
$$

and using $\left(\begin{array}{c}n \\ n+1\end{array}\right)_{q}=\left(\begin{array}{c}n \\ -1\end{array}\right)_{q}=0$, this expression can be written as

$$
\begin{aligned}
D_{q ; w}^{n+1} f(x)= & q^{-n(n-1) / 2}((q-1) x+w)^{-n-1} \\
& \times \sum_{k=0}^{n+1}(-1)^{n-k+1} q^{(n-k+1)(n-k) / 2-k+1}\left(\begin{array}{c}
n \\
k-1
\end{array}\right){ }_{q} f\left(q^{k} x+w \sum_{i=0}^{k-1} q^{i}\right) \\
& -q^{-n(n-1) / 2}((q-1) x+w)^{-n-1} \\
& \times \sum_{k=0}^{n+1}(-1)^{n-k} q^{(n-k)(n-k-1) / 2-k}\left(\begin{array}{l}
n \\
k
\end{array}\right) \underset{q}{f}\left(q^{k} x+w \sum_{i=0}^{k-1} q^{i}\right) .
\end{aligned}
$$


Further simplification yields

$$
\begin{aligned}
D_{q ; w}^{n+1} f(x)= & \frac{q^{-n(n-1) / 2}}{((q-1) x+w)^{n+1}} \sum_{k=0}^{n+1}(-1)^{n-k+1} q^{(n-k-1)(n-k) / 2-k} \\
& \times\left[q^{n-k+1}\left(\begin{array}{c}
n \\
k-1
\end{array}\right)_{q}+\left(\begin{array}{l}
n \\
k
\end{array}\right)\right] f\left(q^{k} x+w \sum_{i=0}^{k-1} q^{i}\right)
\end{aligned}
$$

or simply using (23)

$$
\begin{aligned}
D_{q ; w}^{n+1} f(x)= & \frac{q^{-\frac{1}{2} n(n+1)}}{((q-1) x+w)^{n+1}} \\
& \times \sum_{k=0}^{n+1}(-1)^{n-k+1} q^{\frac{1}{2}(n-k)(n+1-k)}\left(\begin{array}{c}
n+1 \\
k
\end{array}\right) f\left(q_{q}^{k} x+w \sum_{i=0}^{k-1} q^{i}\right)
\end{aligned}
$$

as required.

Remark 1 For arbitrary $n=1,2, \ldots$, the identity

$$
\sum_{k=0}^{n}(-1)^{k} q^{k(k-1) / 2}\left(\begin{array}{c}
n \\
n-k
\end{array}\right)_{q}=0
$$

follows directly from Theorem 3 for the constant function $f(x)$ and the fact that $\sum_{k=0}^{n} A_{n-k}=\sum_{k=0}^{n} A_{k}$. Further, for $0<m<n$,

$$
\sum_{k=0}^{n}(-1)^{k} q^{\frac{1}{2}(n-k)(n-k-1)}\left(\begin{array}{l}
n \\
k
\end{array}\right)_{q}\left(q^{k} x+w \sum_{i=0}^{k-1} q^{i}\right)^{m}=0 .
$$

Remark 2 For arbitrary $n, m=1,2, \ldots$,

$$
\begin{aligned}
& D_{q ; w}^{n} x^{m} \\
& \qquad \begin{cases}0, & \text { if } m<n, \\
\frac{q^{-n(n-1) / 2}}{((1-q) x-w)^{n}} \sum_{k=0}^{n}(-1)^{k} q^{\frac{1}{2}(n-k)(n-k-1)}\left(\begin{array}{l}
n \\
k
\end{array}\right)\left(q^{k} x+w \sum_{i=0}^{k-1} q^{i}\right)^{m}, & \text { if } m \geq n,\end{cases}
\end{aligned}
$$

that follows directly from Theorem 3 for the function $f(x)=x^{m}$.

Definition 1 We define the $n$ th-degree polynomials in the $(q ; w)$-space as

$$
\mathcal{P}_{n}(x)=\prod_{j=0}^{n-1}\left(x-w \sum_{i=0}^{j-1} q^{i}\right)
$$

recalling the empty product and sum: $\prod_{k=0}^{-1} a_{k}=1$ and $\sum_{k=0}^{-1} a_{k}=0$. 
The first few polynomials are given explicitly as follows:

$$
\left\{\begin{array}{l}
\mathcal{P}_{0}(x)=1 \\
\mathcal{P}_{1}(x)=x, \mathcal{P}_{2}(x)=x(x-w) \\
P_{3}(x)=x(x-w)(x-(q+1) w) \\
\mathcal{P}_{4}(x)=x(x-w)(x-(q+1) w)\left(x-\left(q^{2}+q+1\right) w\right), \\
\mathcal{P}_{5}(x)=x(x-w)(x-(q+1) w)\left(x-\left(q^{2}+q+1\right) w\right)\left(x-\left(q^{3}+q^{2}+q+1\right) w\right) .
\end{array}\right.
$$

Theorem 4 The $q$; $w$-derivative of the nth-degree polynomial $\mathcal{P}_{n}(x)$ is given by

$$
\mathcal{P}_{0}(x)=1, \quad D_{q ; w} \mathcal{P}_{n}(x)=\left(\sum_{j=0}^{n-1} q^{j}\right) \mathcal{P}_{n-1}(x), \quad n \geq 1
$$

Proof For arbitrary $n$,

$$
\begin{aligned}
D_{q ; w} \mathcal{P}_{n}(x) & =\frac{\prod_{j=0}^{n-1}\left(q x+w-w \sum_{i=0}^{j-1} q^{i}\right)-\prod_{j=0}^{n-1}\left(x-w \sum_{i=0}^{j-1} q^{i}\right)}{(q-1) x+w} \\
& =\frac{q^{n}\left(x-\frac{w\left(q^{-1}-1\right)}{q-1}\right) \prod_{j=1}^{n-1}\left(x-\frac{w\left(q^{j-1}-1\right)}{q-1}\right)-\prod_{j=0}^{n-1}\left(x-\frac{w\left(q^{j}-1\right)}{q-1}\right)}{(q-1) x+w} .
\end{aligned}
$$

Since $1 \leq j \leq n-1$ implies $0 \leq j-1 \leq n-2$, let $i=j-1$, and it follows that $j=i+1$. Thus

$$
\begin{aligned}
D_{q ; w} \mathcal{P}_{n}(x) & =\frac{q^{n}\left(x-\frac{w\left(q^{-1}-1\right)}{q-1}\right) \prod_{j=0}^{n-2}\left(x-\frac{w\left(q^{j}-1\right)}{q-1}\right)-\prod_{j=0}^{n-1}\left(x-\frac{w\left(q^{j}-1\right)}{q-1}\right)}{(q-1) x+w} \\
& =\prod_{j=0}^{n-2}\left(x-\frac{w\left(q^{j}-1\right)}{q-1}\right) \frac{q^{n}\left(x-\frac{w\left(q^{-1}-1\right)}{q-1}\right)-\left(x-\frac{w\left(q^{n-1}-1\right)}{q-1}\right)}{(q-1) x+w} \\
& =\prod_{j=0}^{n-2}\left(x-\frac{w\left(q^{j}-1\right)}{q-1}\right) \frac{q^{n}-1}{q-1}=\left(\sum_{j=0}^{n-1} q^{j}\right) \prod_{j=0}^{n-2}\left(x-\frac{w\left(q^{j}-1\right)}{q-1}\right) \\
& =\left(\sum_{j=0}^{n-1} q^{j}\right) \mathcal{P}_{n-1}(x)
\end{aligned}
$$

as required.

Theorem 5 In terms of the polynomials $\left\{\mathcal{P}_{n}(x)\right\}_{n=0}$, an arbitrary $r$ degree polynomial $P_{r}(x)$ can be represented by the following expansion:

$$
P_{r}(x)=\sum_{k=0}^{r} \frac{D_{q ; w}^{k} P_{r}(0)}{(1-q)^{-k}(q ; q)_{k}} \mathcal{P}_{k}(x) .
$$

Proof Let $P_{r}(x)$ be the $r^{\text {th }}$ degree polynomial of $x$ that can be written as

$$
P_{r}(x)=\sum_{k=0}^{r} A_{k} \mathcal{P}_{k}(x)
$$


where $A_{k}$ are constants to be determined. Using the direct derivative for $k=0,1,2, \ldots$, followed by the substitution $x=0$ to compute the corresponding $A_{k}$, it is not difficult to prove that

$$
\begin{aligned}
A_{k} & =\frac{D_{q ; w}^{k} P_{r}(0)}{(1+q)\left(1+q+q^{2}\right)\left(1+q+q^{2}+q^{3}\right) \cdots\left(1+q+q^{2}+\cdots+q^{k-1}\right)} \\
& =\frac{D_{q ; w}^{k} P_{r}(0)}{\prod_{j=0}^{k-1}\left(\sum_{i=0}^{j} q^{i}\right)}=\frac{D_{q ; w}^{k} P_{r}(0)}{(1-q)^{-k}(q ; q)_{k}} .
\end{aligned}
$$

Thus,

$$
P_{r}(x)=\sum_{k=0}^{r} \frac{D_{q ; w}^{k} P_{r}(0)}{(1-q)^{-k}(q ; q)_{k}}\left(\prod_{j=0}^{k-1}\left(x-w \sum_{i=0}^{j-1} q^{j}\right)\right) .
$$

For example, the expansions of the polynomials $x^{2}, x^{3}, x^{4}$ in terms of $\left\{\mathcal{P}_{n}(x)\right\}$ are

$$
\begin{aligned}
x^{2}= & w x+x(x-w), \\
x^{3}= & w^{2} x+(q w+2 w) x(x-w)+x(x-w)(x-(q+1) w), \\
x^{4}= & w^{3} x+\left(q^{2}+3 q+3\right) w^{2} x(x-w) \\
& +\left(q^{2}+2 q+3\right) w x(x-w)(x-(1+q) w) \\
& +x(x-w)(x-(1+q) w)\left(x-\left(1+q+q^{2}\right) w\right) .
\end{aligned}
$$

Corollary 1 If $P_{r}(x)$ is an r-degree polynomial, then

$$
D_{q ; w}^{n} P_{r}(x)=0, \quad \text { for all } n \geq r+1 \text {. }
$$

\section{$3 q_{\text {; w }}$ AIM for Hahn difference equations}

Several papers over the past few years have addressed the theory of the linear Hahn difference equations [17, 35-40]. The existence and uniqueness theorems of solutions of linear Hahn difference equations are given in [35]. In [10], Annaby, Hamza, and Aldwoah solved the first order linear Hahn difference equations with constant coefficients.

\subsection{First-order linear Hahn difference equations}

We consider in this subsection the first-order Hahn difference equation with variable coefficients

$$
D_{q ; w} y(x)=\alpha(x) y(x)+\beta(x), \quad 0<w, 0<q<1,
$$

where $\alpha(x)$ and $\beta(x)$ are arbitrary functions. Note that, using Hahn's operator definition (1), equation (33) can be written as

$$
y(q x+w)=(1+((q-1) x+w) \alpha(x)) y(x)+((q-1) x+w) \beta(x) .
$$


Theorem 6 For $0<q<1$, the general solution of the q; $w$-difference equation of the firstorder Hahn difference equation (33) or (34) is

$$
\begin{aligned}
y(x)= & \frac{c}{\prod_{k=0}^{\infty}\left(1+q^{k}((q-1) x+w) \alpha\left(w \sum_{i=0}^{k-1} q^{i}+x q^{k}\right)\right)} \\
& -\sum_{k=0}^{\infty} \frac{q^{k}((q-1) x+w) \beta\left(w \sum_{i=0}^{k-1} q^{i}+x q^{k}\right)}{\prod_{j=0}^{k}\left(1+q^{j}((q-1) x+w) \alpha\left(w \sum_{i=0}^{j-1} q^{i}+x q^{j}\right)\right)},
\end{aligned}
$$

where $c(q x+w)=c(x)$ is a periodic (constant) function.

Proof We prove this theorem by direct substitution. By the given solution

$$
\begin{aligned}
y(q x+w)= & \frac{c}{\prod_{k=0}^{\infty}\left(1+q^{k+1}((q-1) x+w) \alpha\left(w \sum_{i=0}^{k} q^{i}+q^{k+1} x\right)\right.} \\
& -\sum_{k=0}^{\infty} \frac{q^{k+1}((q-1) x+w) \beta\left(w \sum_{i=0}^{k} q^{i}+q^{k+1} x\right)}{\prod_{j=0}^{k}\left(1+q^{j+1}((q-1) x+w) \alpha\left(w \sum_{i=0}^{j} q^{i}+q^{j+1} x\right)\right)},
\end{aligned}
$$

shift the indices of the finite products

$$
\begin{aligned}
y(q x+w)= & \frac{c}{\prod_{k=1}^{\infty}\left(1+q^{k}((q-1) x+w) \alpha\left(w \sum_{i=0}^{k-1} q^{i}+q^{k} x\right)\right.} \\
& -\sum_{k=1}^{\infty} \frac{q^{k}((q-1) x+w) \beta\left(w \sum_{i=0}^{k-1} q^{i}+q^{k} x\right)}{\prod_{j=0}^{k-1}\left(1+q^{j+1}((q-1) x+w) \alpha\left(w \sum_{i=0}^{j} q^{i}+q^{j+1} x\right)\right)},
\end{aligned}
$$

which can be written as

$$
\begin{aligned}
y(q x+w)= & \frac{(1+((q-1) x+w) \alpha(x)) c}{\prod_{k=0}^{\infty}\left(1+q^{k}((q-1) x+w) \alpha\left(w \sum_{i=0}^{k-1} q^{i}+q^{k} x\right)\right.} \\
& -\sum_{k=1}^{\infty} \frac{q^{k}((q-1) x+w) \beta\left(w \sum_{i=0}^{k-1} q^{i}+q^{k} x\right)}{\prod_{j=0}^{k-1}\left(1+q^{j+1}((q-1) x+w) \alpha\left(w \sum_{i=0}^{j} q^{i}+q^{j+1} x\right)\right)} .
\end{aligned}
$$

Using the given solution again, it follows that

$$
\begin{aligned}
y(q x+w)= & (1+((q-1) x+w) \alpha(x)) \\
& \times\left(y(x)+\sum_{k=0}^{\infty} \frac{q^{k}((q-1) x+w) \beta\left(w \sum_{i=0}^{k-1} q^{i}+q^{k} x\right)}{\prod_{j=0}^{k}\left(q^{j}((q-1) x+w) \alpha\left(w \sum_{i=0}^{j-1} q^{i}+x q^{j}\right)+1\right)}\right) \\
& -\sum_{k=1}^{\infty} \frac{q^{k}((q-1) x+w) \beta\left(w \sum_{i=0}^{k-1} q^{i}+q^{k} x\right)}{\prod_{j=0}^{k-1}\left(1+q^{j+1}((q-1) x+w) \alpha\left(w \sum_{i=0}^{j} q^{i}+q^{j+1} x\right)\right)},
\end{aligned}
$$

which simplifies to

$$
y(q x+w)=(1+((q-1) x+w) \alpha(x)) y(x)+((q-1) x+w) \beta(x) .
$$

Remark 3 For $0<q<1$, a solution of the homogeneous $q$; $w$-difference equation

$$
D_{q ; w} y(x)=\alpha(x) y(x)
$$


up to a multiplicative constant is

$$
y(x)=\prod_{k=0}^{\infty}\left(1+q^{k}((q-1) x+w) \alpha\left(w \sum_{i=0}^{k-1} q^{i}+x q^{k}\right)\right)^{-1} .
$$

Remark 4 The exact solution of the limiting case $w \rightarrow 0$ [26]

$$
D_{q} y(x)=\alpha(x) y(x)+\beta(x), \quad 0<q<1,
$$

is

$$
y(x)=\frac{c}{\prod_{k=0}^{\infty}\left(1-q^{k}(1-q) x \alpha\left(q^{k} x\right)\right)}-\sum_{k=0}^{\infty} \frac{q^{k}(q-1) x \beta\left(q^{k} x\right)}{\prod_{j=0}^{k}\left(1+q^{j}(q-1) x \alpha\left(q^{j} x\right)\right)}
$$

as developed earlier in [26].

Example 1 The solution of the first-order Hahn difference equation

$$
D_{q ; w} y(x)=y(x), \quad 0<q<1,
$$

up to some multiplicative constant is

$$
y(x)=\frac{1}{\prod_{k=0}^{\infty}\left(1+q^{k}((q-1) x+w)\right)}=\frac{1}{((1-q) x-w ; q)_{\infty}},
$$

which is the $q ; w$-exponential function [27, Theorem 12.2.6] as expected for this difference equation.

Example 2 The solution of the first-order Hahn difference equation

$$
D_{q ; w} y(x)=a y(x)+b, \quad 0<q<1,
$$

where $a$ and $b$ are real constants, is

$$
y(x)=\frac{c}{(a((1-q) x-w) ; q)_{\infty}}-\sum_{k=0}^{\infty} \frac{b q^{k}((q-1) x+w)}{(a((1-q) x-w) ; q)_{k+1}} .
$$

\section{2 q:w AIM for the second-order Hahn difference equations}

In this section, we consider the second-order Hahn difference equation

$$
y\left(q^{2} x+(1+q) w\right)=\alpha(x) y(q x+w)+\beta(x) y(x)
$$

where $\alpha(x)$ and $\beta(x)$ are $(q ; w)$-differentiable functions. Using the easily proved identity

$$
D_{q ; w}^{2} f(x)=\frac{f\left(q^{2} x+(1+q) w\right)-(1+q) f(q x+w)+q f(x)}{q((q-1) x+w)^{2}},
$$


equation (38) can be written equivalently as

$$
D_{q ; w}^{2} y(x)=\lambda_{0}(x) D_{q ; w} y(x)+s_{0}(x) y(x),
$$

where

$$
\lambda_{0}(x)=\frac{\alpha(x)-q-1}{q((q-1) x+w)}, \quad s_{0}(x)=\frac{\alpha(x)+\beta(x)-1}{q((q-1) x+w)^{2}} .
$$

Although these two forms (38) and (40) are equivalent, we shall focus our attention on the form (40) to investigate the solutions of the second-order linear Hahn difference equation (40) with variable coefficients.

\subsubsection{General solution}

Acting by the Hahn difference operator $D_{q ; w}$ on equation (40) yields

$$
D_{q ; w}^{3} y(x)=D_{q ; w}\left(D_{q ; w}^{2} y(x)\right)=D_{q ; w}\left(\lambda_{0}(x) D_{q ; w} y(x)\right)+D_{q ; w}\left(s_{0}(x) y(x)\right) .
$$

However, by means of the product rule (17), it easily follows that

$$
D_{q ; w}^{3} y(x)=\lambda_{1}(x) D_{q ; w} y(x)+s_{1}(x) y(x),
$$

where

$$
\left\{\begin{array}{l}
\lambda_{1}(x)=D_{q ; w} \lambda_{0}(x)+\lambda_{0}(q x+w) \lambda_{0}(x)+s_{0}(q x+w) \\
s_{1}(x)=D_{q ; w} s_{0}(x)+\lambda_{0}(q x+w) s_{0}(x)
\end{array}\right.
$$

Similarly, applying the Hahn difference once more yields

$$
D_{q ; w}^{4} y(x)=\lambda_{2}(x) D_{q ; w} y(x)+s_{2}(x) y(x)
$$

where

$$
\left\{\begin{array}{l}
\lambda_{2}(x)=D_{q ; w} \lambda_{1}(x)+\lambda_{1}(q x+w) \lambda_{0}(x)+s_{1}(q x+w) \\
s_{2}(x)=D_{q ; w} s_{1}(x)+\lambda_{1}(q x+w) s_{0}(x)
\end{array}\right.
$$

In general, we may apply this process to obtain, for $n=1,2, \ldots$, that

$$
\begin{aligned}
& D_{q ; w}^{n+1} y(x)=\lambda_{n-1}(x) D_{q ; w} y(x)+s_{n-1}(x) y(x), \\
& D_{q ; w}^{n+2} y(x)=\lambda_{n}(x) D_{q ; w} y(x)+s_{n}(x) y(x)
\end{aligned}
$$

where

$$
\left\{\begin{array}{l}
\lambda_{n}(x)=D_{q ; w} \lambda_{n-1}(x)+\lambda_{n-1}(q x+w) \lambda_{0}(x)+s_{n-1}(q x+w) \\
s_{n}(x)=D_{q ; w} s_{n-1}(x)+\lambda_{n-1}(q x+w) s_{0}(x)
\end{array}\right.
$$


The general form (43) can be proved by induction on $n$. For $n=1$, the identity is true by construction. Assume it is true for $n+1$, then for $n+2$

$$
\begin{aligned}
D_{q ; w}^{n+2} y(x) & =D_{q ; w}\left(D_{q ; w}^{n+1} y(x)\right) \\
& =D_{q ; w}\left(\lambda_{n-1}(x) D_{q ; w} y(x)+s_{n-1}(x) y(x)\right),
\end{aligned}
$$

and by the product rule (17),

$$
\begin{aligned}
D_{q ; w}^{n+2} y(x)= & \lambda_{n-1}(q x+w) D_{q ; w}^{2} y(x)+D_{q ; w} y(x) D_{q ; w} \lambda_{n-1}(x) \\
& +s_{n-1}(q x+w) D_{q ; w} y(x)+y(x) D_{q ; w} s_{n-1}(x) .
\end{aligned}
$$

Using (40) and simplifying, we obtain

$$
\begin{aligned}
D_{q ; w}^{n+2} y(x)= & \left(D_{q ; w} \lambda_{n-1}(x)+\lambda_{n-1}(q x+w) \lambda_{0}(x)+s_{n-1}(q x+w)\right) D_{q ; w} y(x) \\
& +\left(D_{q ; w} s_{n-1}(x)+\lambda_{n-1}(x) s_{0}(x)\right) y(x) \\
= & \lambda_{n}(x) D_{q ; w} y(x)+s_{n}(x) y(x)
\end{aligned}
$$

as expected. Now dividing (42) and (41), we obtain

$$
\frac{D_{q ; w}^{n+2} y(x)}{D_{q ; w}^{n+1} y(x)}=\frac{\lambda_{n}(x)\left[D_{q ; w} y(x)+\frac{s_{n}(x)}{\lambda_{n}(x)} y(x)\right]}{\lambda_{n-1}(x)\left[D_{q ; w} y(x)+\frac{s_{n-1}(x)}{\lambda_{n-1}(x)} y(x)\right]} .
$$

Thus, if for some $n \geq 1$ the so-called terminating condition

$$
\frac{s_{n}(x)}{\lambda_{n}(x)}=\frac{s_{n-1}(x)}{\lambda_{n-1}(x)}
$$

is satisfied, then it follows that

$$
\frac{D_{q ; w}^{n+2} y(x)}{D_{q ; w}^{n+1} y(x)}=\frac{\lambda_{n}(x)}{\lambda_{n-1}(x)}
$$

This is easily written as a first-order difference equation in $D_{q ; w}^{n+1} y(x)$

$$
D_{q ; w}\left[D_{q ; w}^{n+1} y(x)\right]=\frac{\lambda_{n}(x)}{\lambda_{n-1}(x)} D_{q ; w}^{n+1} y(x)
$$

with a solution for $0<q<1$ given by, see Theorem 6 ,

$$
D_{q ; w}^{n+1} y(x)=\frac{c_{2}}{\prod_{k=0}^{\infty}\left[1+q^{k}(w+(q-1) x) \frac{\lambda_{n}\left(q^{k} x+w \sum_{i=0}^{k-1} q^{i}\right)}{\lambda_{n-1}\left(q^{k} x+w \sum_{i=0}^{k-1} q^{i}\right)}\right]}
$$

for some periodic (constant) function $c_{2}$. Equation (41) reduces to

$$
\begin{aligned}
D_{q ; w} y(x)= & -\frac{s_{n-1}(x)}{\lambda_{n-1}(x)} y(x) \\
& +\frac{1}{\lambda_{n-1}(x)} \frac{c_{2}}{\prod_{i=0}^{\infty}\left[1+q^{i}(w+(q-1) x) \frac{\lambda_{n}\left(q^{i} x+w \sum_{l=0}^{i-1} q^{l}\right)}{\lambda_{n-1}\left(q^{i} x+w \sum_{l=0}^{i-1} q^{l}\right)}\right]},
\end{aligned}
$$


with the general solution given by

$$
\begin{aligned}
y(x)= & \frac{c_{1}}{\prod_{k=0}^{\infty}\left[1+q^{k}((1-q) x-w) \frac{s_{n-1}\left(x_{k}\right)}{\lambda_{n-1}\left(x_{k}\right)}\right]} \\
& -\sum_{i=0}^{\infty} \frac{\frac{q^{i}((q-1) x+w)}{\lambda_{n-1}\left(x_{i}\right)}}{\prod_{j=0}^{i}\left[1+q^{j}((1-q) x-w) \frac{s_{n-1}\left(x_{j}\right)}{\lambda_{n-1}\left(x_{j}\right)}\right]} \\
& \times \frac{c_{2}}{\prod_{j=0}^{\infty}\left[1+q^{i+j}((q-1) x+w) \frac{\lambda_{n}\left(q^{i+j} x+w \sum_{l=0}^{i+j-1} q^{l}\right)}{\lambda_{n-1}\left(q^{i+j} x+w \sum_{l=0}^{i+j-1} q^{l}\right)}\right]},
\end{aligned}
$$

where $x_{k}=q^{k} x+w \sum_{j=0}^{k-1} q^{j}$ and $c_{i}, i=1,2$, are periodic functions with the property that $D_{q ; w} c_{i}=0, i=1,2$. It is a straightforward exercise to show that the two solutions are linearly independent by proving that the $(q ; w)$-Casorati determinant

$$
\left|\begin{array}{ll}
y_{1}(x) & y_{1}(q x+w) \\
y_{2}(x) & y_{2}(q x+w)
\end{array}\right|
$$

does not vanish. We summarize this result in the following theorem.

Theorem 7 A solution of the second-order Hahn linear difference equation

$$
D_{q: w}^{2} y(x)=\lambda_{0}(x) D_{q: w} y(x)+s_{0}(x) y(x)
$$

is given by

$$
y_{n}(x)=\prod_{k=0}^{\infty}\left[1+q^{k}((1-q) x-w) \frac{s_{n-1}\left(x_{k}\right)}{\lambda_{n-1}\left(x_{k}\right)}\right]^{-1}
$$

provided that, for some $n>0$,

$$
\frac{s_{n}(x)}{\lambda_{n}(x)}=\frac{s_{n-1}(x)}{\lambda_{n-1}(x)} \quad \text { or } \quad \delta_{n}(x) \equiv \lambda_{n}(x) s_{n-1}(x)-\lambda_{n-1}(x) s_{n}(x)=0
$$

where

$$
\left\{\begin{array}{l}
\lambda_{n}(x)=D_{q ; w} \lambda_{n-1}(x)+\lambda_{n-1}(q x+w) \lambda_{0}(x)+s_{n-1}(q x+w), \\
s_{n}(x)=D_{q ; w} s_{n-1}(x)+\lambda_{n-1}(q x+w) s_{0}(x) .
\end{array}\right.
$$

Remark 5 In general, the terminating condition (45) is satisfied for $n \rightarrow \infty$. As discussed and proved in the next section, the condition holds for finite $n$ if the difference equation can be solved exactly by a polynomial of at most $n$ degree. As far as we know, it is not possible to predict whether such a condition will hold for specific $n$ without an experiment.

\subsubsection{A criterion for polynomial solutions}

We give in Theorems 8 and 9 below the necessary and sufficient conditions respectively for a second order linear Hahn difference equation (40) to have a polynomial solution. 
Theorem 8 If a solution of the difference equation

$$
D_{q: w}^{2} y(x)=\lambda_{0}(x) D_{q: w} y(x)+s_{0}(x) y(x)
$$

is a polynomial of at most of degree $n$, then $\delta_{n}(x)=0$.

Proof Multiplying equation (41) by $\lambda_{n}(x)$ and (42) by $\lambda_{n-1}(x)$ yields

$$
\begin{aligned}
& \lambda_{n}(x) D_{q ; w}^{n+1} y(x)=\lambda_{n}(x) \lambda_{n-1}(x) D_{q ; w} y(x)+\lambda_{n}(x) s_{n-1}(x) y(x), \\
& \lambda_{n-1}(x) D_{q ; w}^{n+2} y(x)=\lambda_{n-1}(x) \lambda_{n}(x) D_{q ; w} y(x)+\lambda_{n-1}(x) s_{n}(x) y(x) .
\end{aligned}
$$

Subtracting these two equations, it follows that

$$
\begin{aligned}
\lambda_{n}(x) D_{q ; w}^{n+1} y(x)-\lambda_{n-1}(x) D_{q ; w}^{n+2} y(x) & =\left[\lambda_{n}(x) s_{n-1}(x)-\lambda_{n-1}(x) s_{n}(x)\right] y(x) \\
& =\delta_{n}(x) y(x) .
\end{aligned}
$$

Consequently, if $y(x)$ is a polynomial of at most $n$, it follows by Corollary 1 that $D_{q ; w}^{n+2} y(x)=$ $D_{q ; w}^{n+1} y(x)=0$; and $\delta_{n}(x)=0$.

Lemma 3 If

$$
y(x)=\frac{1}{\prod_{k=0}^{\infty}\left[1+q^{k}((1-q) x-w) \frac{s_{n-1}\left(q^{k} x+w \sum_{i=0}^{k-1} q^{i}\right)}{\lambda_{n-1}\left(q^{k} x+w \sum_{i=0}^{k-1} q^{i}\right)},\right.},
$$

then

$$
D_{q ; w} y(x)=-\frac{s_{n-1}(x)}{\lambda_{n-1}(x)} \times y(x) .
$$

Proof It is not difficult to show, by shifting the indices of the product definitions of $y(x)$ and $y(q x+w)$, that the difference $y(q x+w)-y(x)$ reduces to

$$
\begin{aligned}
y(q x+w)-y(x)= & \prod_{k=1}^{\infty}\left[1+q^{k}((1-q) x-w) \frac{s_{n-1}\left(q^{k} x+w \sum_{i=0}^{k-1} q^{i}\right)}{\lambda_{n-1}\left(q^{k} x+w \sum_{i=0}^{k-1} q^{i}\right)}\right]^{-1} \\
& -\prod_{k=0}^{\infty}\left[1+q^{k}((1-q) x-w) \frac{s_{n-1}\left(q^{k} x+w \sum_{i=0}^{k-1} q^{i}\right)}{\lambda_{n-1}\left(q^{k} x+w \sum_{i=0}^{k-1} q^{i}\right)}\right]^{-1} .
\end{aligned}
$$

Thus, by definition of the difference operator,

$$
\begin{aligned}
D_{q ; w} y(x) & =\frac{y(q x+w)-y(x)}{(q-1) x+w} \\
& =y(x) \times \frac{\left[1+((1-q) x-w) \frac{s_{n-1}(x)}{\lambda_{n-1}(x)}\right]-1}{(q-1) x+w}=-y(x) \times \frac{s_{n-1}(x)}{\lambda_{n-1}(x)} .
\end{aligned}
$$

Theorem 9 If $\delta_{n}=0$ and $\lambda_{n-1}(x) \neq 0$, then the solution of the difference equation

$$
D_{q: w}^{2} y(x)=\lambda_{0}(x) D_{q: w} y(x)+s_{0}(x) y(x)
$$

is a polynomial of at most degree $n$. 
Proof Given $\delta_{n}(x)=0$, it follows by Theorem 6 that

$$
y(x)=\frac{1}{\prod_{k=0}^{\infty}\left[1+q^{k}((1-q) x-w) \frac{s_{n-1}\left(q^{k} x+w \sum_{i=0}^{k-1} q^{i}\right)}{\lambda_{n-1}\left(q^{k} x+w \sum_{i=0}^{k-1} q^{i}\right)},\right.},
$$

which allows one to evaluate $D_{q ; w} y(x)$ as, see Lemma 3,

$$
D_{q ; w} y(x)=-y(x) \times \frac{s_{n-1}(x)}{\lambda_{n-1}(x)} .
$$

Thus, using equation (41), we have

$$
\begin{aligned}
D_{q ; w}^{n+1} y(x) & =\lambda_{n-1}(x) D_{q ; w} y(x)+s_{n-1}(x) y(x) \\
& =\left(-\lambda_{n-1}(x) \frac{s_{n-1}(x)}{\lambda_{n-1}(x)}+s_{n-1}(x)\right) y(x)=0 .
\end{aligned}
$$

Theorem 10 Let $\lambda_{n}(x)$ and $s_{n}(x)$ be as in (44), and set $\delta_{n}(x)=\lambda_{n}(x) s_{n-1}(x)-\lambda_{n-1}(x) s_{n}(x)$. If $\delta_{n}(x)=0$, then $\delta_{n^{\prime}}(x)=0$ for all $n^{\prime} \geq n+1$.

Proof Note that, by the sequence relations of $\lambda_{n}(x)$ and $s_{n}(x)$, we have

$$
\begin{aligned}
\delta_{n+1}(x)= & \lambda_{n+1}(x) s_{n}(x)-\lambda_{n}(x) s_{n+1}(x) \\
= & s_{n}(x) D_{q ; w} \lambda_{n}(x)+s_{n}(x) \lambda_{n}(q x+w) \lambda_{0}(x)+s_{n}(q x+w) s_{n}(x) \\
& -\lambda_{n}(x) D_{q ; w} s_{n}(x)-\lambda_{n}(x) \lambda_{n}(q x+w) s_{0}(x) .
\end{aligned}
$$

This equation can be easily written as

$$
\begin{aligned}
\delta_{n+1}(x)= & s_{n}(q x+w) s_{n}(x)\left(\frac{s_{n}(x) D_{q ; w} \lambda_{n}(x)-\lambda_{n}(x) D_{q ; w} s_{n}(x)}{s_{n}(q x+w) s_{n}(x)}\right) \\
& +s_{n}(q x+w) s_{n}(x)+s_{n}(x) \lambda_{n}(q x+w) \lambda_{0}(x)-\lambda_{n}(x) \lambda_{n}(q x+w) s_{0}(x),
\end{aligned}
$$

where the quotient rule yields

$$
\begin{aligned}
\delta_{n+1}(x)= & s_{n}(q x+w) s_{n}(x) D_{q ; w}\left(\frac{\lambda_{n}(x)}{s_{n}(x)}\right) \\
& +s_{n}(q x+w) s_{n}(x)+s_{n}(x) \lambda_{n}(q x+w) \lambda_{0}(x)-\lambda_{n}(x) \lambda_{n}(q x+w) s_{0}(x) .
\end{aligned}
$$

Further simplification implies

$$
\begin{aligned}
\delta_{n+1}(x)= & s_{n}(q x+w) s_{n}(x) \\
& \times\left[D_{q ; w}\left(\frac{\lambda_{n}(x)}{s_{n}(x)}\right)+1+\frac{\lambda_{n}(q x+w)}{s_{n}(q x+w)} \lambda_{0}(x)-\frac{\lambda_{n}(x) \lambda_{n}(q x+w) s_{0}(x)}{s_{n}(x) s_{n}(q x+w)}\right] .
\end{aligned}
$$

Using the terminating condition, it follows that

$$
\begin{aligned}
\delta_{n+1}(x)= & s_{n}(q x+w) s_{n}(x) \\
& \times\left[D_{q ; w}\left(\frac{\lambda_{n-1}(x)}{s_{n-1}(x)}\right)+1+\frac{\lambda_{n-1}(q x+w)}{s_{n-1}(q x+w)} \lambda_{0}(x)-\frac{\lambda_{n-1}(x) \lambda_{n-1}(q x+w) s_{0}(x)}{s_{n-1}(x) s_{n-1}(q x+w)}\right],
\end{aligned}
$$


which implies

$$
\begin{aligned}
\delta_{n+1}(x)= & s_{n}(q x+w) s_{n}(x)\left[\frac{s_{n-1} D_{q ; w} \lambda_{n-1}(x)-\lambda_{n-1}(x) D_{n-1} s_{n-1}(x)}{s_{n-1}(x) s_{n-1}(q x+w)}\right. \\
& \left.+1+\frac{\lambda_{n-1}(q x+w)}{s_{n-1}(q x+w)} \lambda_{0}(x)-\frac{\lambda_{n-1}(x) \lambda_{n-1}(q x+w) s_{0}(x)}{s_{n-1}(x) s_{n-1}(q x+w)}\right] \\
= & s_{n}(q x+w) s_{n}(x)\left[\frac{s_{n-1}(x) D_{q ; w} \lambda_{n-1}(x)-\lambda_{n-1}(x) D_{q ; w} s_{n-1}(x)}{s_{n-1}(x) s_{n-1}(q x+w)}\right. \\
& +\frac{s_{n-1}(x) s_{n-1}(q x+w)}{s_{n-1}(x) s_{n-1}(q x+w)}+\frac{s_{n-1}(x) \lambda_{n-1}(q x+w)}{s_{n-1}(x) s_{n-1}(q x+w)} \lambda_{0}(x) \\
& \left.-\frac{\lambda_{n-1}(x) \lambda_{n-1}(q x+w) s_{0}(x)}{s_{n-1}(x) s_{n-1}(q x+w)}\right] .
\end{aligned}
$$

Finally, we have

$$
\begin{aligned}
\delta_{n+1}(x)= & s_{n}(q x+w) s_{n}(x) \\
& \times\left[\frac{s_{n-1}(x)\left(D_{q ; w} \lambda_{n-1}(x)+\lambda_{n-1}(q x+w) \lambda_{0}(x)+\lambda_{n-1}(q x+w)\right)}{s_{n-1}(x) s_{n-1}(q x+w)}\right. \\
& \left.-\frac{\lambda_{n-1}(x)\left(D_{n-1} s_{n-1}(x)+\lambda_{n-1}(q x+w) s_{0}(x)\right)}{s_{n-1}(x) s_{n-1}(q x+w)}\right] \\
= & s_{n}(q x+w) s_{n}(x)\left[\frac{s_{n-1}(x) \lambda_{n}(x)-\lambda_{n-1}(x) s_{n}(x)}{s_{n-1}(x) s_{n-1}(q x+w)}\right]=0
\end{aligned}
$$

because by assumption $\delta_{n}=0$. Since $\delta_{n+1}=0, \delta_{n+2}=0$, and so on. Therefore, $\delta_{n^{\prime}}=0$ for all $n^{\prime} \geq n+1$, which completes the proof.

\section{Examples}

We consider first the Hahn difference equation with constant coefficients

$$
D_{q ; w}^{2} y(x)=\lambda_{0} D_{q ; w} y(x)+s_{0} y(x)
$$

where $a$ and $b$ are real constants. Although it is clear, for all $n \geq 1$, that $D_{q ; w} \lambda_{n-1}=$ $D_{q ; w} s_{n-1}=0$, the terminating condition $\delta_{n}$ does not equal 0 for any fixed $n$. The ${ }_{q ; w}$ AIM sequences $\lambda_{n}=\lambda_{n-1} \lambda_{0}+s_{n-1}$ and $s_{n}=\lambda_{n-1} s_{0}$, however, yield

$$
\frac{s_{n}}{\lambda_{n}}=\frac{\lambda_{n-1} s_{0}}{\lambda_{n-1} \lambda_{0}+s_{n-1}} .
$$

Thus, as $n \rightarrow \infty$, the terminating condition reads

$$
\frac{s_{\infty}}{\lambda_{\infty}}=\frac{s_{0}}{\lambda_{0}+\frac{s_{\infty}}{\lambda_{\infty}}} .
$$

This is a quadratic equation with solution given by

$$
\frac{s_{\infty}}{\lambda_{\infty}}=\frac{-\lambda_{0} \pm \sqrt{\lambda_{0}^{2}+4 s_{0}}}{2} .
$$


The two linearly independent solutions are then

$$
\begin{aligned}
y_{ \pm}(x) & =\prod_{i=0}^{\infty}\left[1-q^{i}((q-1) x+w) \frac{-\lambda_{0} \pm \sqrt{\lambda_{0}^{2}+4 s_{0}}}{2}\right]^{-1} \\
& =\frac{1}{\left(\frac{-2 \lambda_{0} \pm \sqrt{\lambda_{0}^{2}+4 s_{0}}}{2}(w+(q-1) x) ; q\right)_{\infty}} .
\end{aligned}
$$

As a second example, we consider the $q$; $w$-hypergeometric equation [28]

$$
D_{q ; w}^{2} y(x)=\underbrace{-\frac{2 \varepsilon x+\gamma}{e x^{2}+2 f x+g}}_{\lambda_{0}(x)} D_{q ; w} y(x)-\underbrace{-\frac{\tau}{e x^{2}+2 f x+g}}_{s_{0}(x)} y(x),
$$

with the constant coefficients $\varepsilon, \gamma, e, f, g$, and $\tau$.

Considering the terminating condition for $n=1$,

$$
\delta_{1}=\lambda_{1} s_{0}-s_{1} \lambda_{0}=\tau(2 \varepsilon+\tau) \equiv 0 \quad \text { if } \tau=0,-2 \varepsilon .
$$

Thus, for $\tau=0$, the polynomial solution $y(x)=P_{n}(x)$ is $P_{0}(x)=1$.

For $\tau=-2 \varepsilon$, the exact polynomial solution, up to a multiplicative constant, is

$$
P_{1}(x)=\lim _{m \rightarrow \infty} \frac{1}{\prod_{k=0}^{m}\left[1+q^{k}((1-q) x-w) \frac{s_{0}\left(q^{k} x+w \sum_{i=0}^{k-1} q^{i}\right)}{\lambda_{0}\left(q^{k} x+w \sum_{i=0}^{k-1} q^{i}\right)}\right.}=x+\frac{\gamma}{2 \varepsilon} \text {. }
$$

The second iteration $n=2$ yields

$$
\delta_{2}=\lambda_{2} s_{1}-s_{2} \lambda_{1}=\tau(2 \varepsilon+\tau)((1+q)(e+2 \varepsilon)+\tau),
$$

where

$$
\left\{\begin{array}{l}
\lambda_{1}(x)=\frac{2 \varepsilon \gamma w+\gamma(2 f+\gamma+e w)-g(2 \varepsilon+\tau)+((e+2 \varepsilon)(\gamma+\gamma q+2 \varepsilon w)-2 f \tau) x+(2 \varepsilon(e+2 \varepsilon) q-e \tau) x^{2}}{(g+x(2 f+e x)(g+(w+q x)(2 f+e(w+q x)))} \\
s_{1}(x)=\frac{\tau(2 f+\gamma+(e+2 \varepsilon) w)+((1+q) e+2 \varepsilon q) \tau x}{(g+x(2 f+e x))(g+(w+q x)(2 f+e(w+q x)))},
\end{array}\right.
$$

then $\delta_{2}=0$ if

$$
\tau=0, \quad-2 \varepsilon, \quad-(1+q)(e+2 \varepsilon) .
$$

For $\tau=-(1+q)(e+2 \varepsilon)$, the second-order polynomial solution is then

$$
\begin{aligned}
P_{2}(x)= & \lim _{m \rightarrow \infty} \frac{1}{\prod_{k=0}^{m}\left[1+q^{k}((1-q) x-w) \frac{s_{1}\left(q^{k} x+w \sum_{i=0}^{k-1} q^{i}\right)}{\lambda_{1}\left(q^{k} x+w \sum_{i=0}^{k-1} q^{i}\right)}\right]} \\
= & x^{2}+\frac{(2 f+\gamma)(1+q)+2 \varepsilon w}{(1+q) e+2 \varepsilon q} x \\
& +\frac{2 \varepsilon g q+\gamma(2 f+\gamma+2 \varepsilon w)+e((1+q) g+\gamma w)}{(e+2 \varepsilon)((1+q) e+2 \varepsilon q)} .
\end{aligned}
$$


Next, the third iteration $n=3$ yields

$$
\delta_{3}=\tau(2 \varepsilon+\tau)((q+1)(e+2 \varepsilon)+\tau)\left(\left(q^{2}+q+1\right)((1+q) e+2 \varepsilon)+\tau\right) .
$$

Thus, for

$$
\tau=-\left(1+q+q^{2}\right)((1+q) e+2 \varepsilon)
$$

the third-order polynomial reads as follows:

$$
\begin{aligned}
P_{3}(x)= & x^{3}+\frac{\tau_{2}}{q\left(2 \varepsilon q+e(1+q)^{2}\right)} x^{2}+\frac{\tau_{1}}{\left(q\left(e(q+1)^{2}+2 \varepsilon q\right)\left(e \sum_{i=0}^{2} q^{i}+2 \varepsilon q\right)\right)} x \\
& +\frac{\tau_{0}}{\left(q((1+q) e+2 \varepsilon)\left(2 \varepsilon q+e(1+q)^{2}\right)\left(2 \varepsilon q+e\left(1+q+q^{2}\right)\right)\right)}
\end{aligned}
$$

where

$$
\begin{aligned}
\tau_{2}= & (\gamma+2 f(1+q)) \sum_{i=0}^{2} q^{i}+(2(1+2 q) \varepsilon+e(1+q)(2+q)) w, \\
\tau_{1}= & \sum_{i=0}^{2} q^{i}\left(\gamma^{2}+4 f^{2}(1+q)+2 f \gamma(2+q)+g q\left(2 \varepsilon q+e(1+q)^{2}\right)\right) \\
& +2((1+q) e+2 \varepsilon) \sum_{i=0}^{2} q^{i}(\gamma+f(2+q)) w+2 \varepsilon(2 \varepsilon(1+q)+e(2+q(2+q))) w^{2}, \\
\tau_{0}= & 4 f^{2} \gamma(1+q)+\gamma\left(\gamma^{2}+2 \varepsilon g q(1+2 q)\right)+2 \varepsilon\left(2 \varepsilon g q(1+q)^{2}+\gamma^{2}(2+q)\right) w \\
& +4 \varepsilon^{2} \gamma(1+q) w^{2}+e^{2}(1+q) w\left(g(1+q)(2+q)\left(1+q+q^{2}\right)+\gamma(2+q(2+q)) w\right) \\
& +2 f\left(2 \varepsilon g q(1+q)^{2}+\gamma^{2}(2+q)+2 \varepsilon \gamma(2+q(2+q)) w\right) \\
& +e\left(g(1+q)\left(\gamma+2(1+q)\left(\gamma q+f\left(1+q+q^{2}\right)\right)\right)\right. \\
& +\left(2 \varepsilon g(1+q)^{3}(1+2 q)+\gamma(2 f(1+q)(3+q(2+q))\right. \\
& \left.+\gamma(3+q(3+q)))) w+2 \varepsilon \gamma(3+2 q(2+q)) w^{2}\right) .
\end{aligned}
$$

In general, it is not difficult to show that

$$
\delta_{n}=\prod_{k=0}^{n}\left(\left(\sum_{j=0}^{k-1} q^{j}\right)\left(2 \varepsilon+e \sum_{j=0}^{k-2} q^{j}\right)+\tau\right) \equiv 0,
$$

and the necessary condition for the existence of the $n$-degree polynomial solution (see also [28, formula 10.1.1]) of the ( $q ; w)$-hypergeometric equation $(54)$, for $n=1,2, \ldots$, is that

$$
\tau_{n}=-\left(\sum_{j=0}^{n-1} q^{j}\right)\left(2 \varepsilon+e \sum_{j=0}^{n-2} q^{j}\right) .
$$

With the ${ }_{q ; w}$ AIM technique under disposal, we hope it will be useful to study the analytic and approximate solutions of the $q$-difference equation. It will also be a practical tool 
in the construction of $q$-orthogonal polynomials and deepen our understanding of their mathematical properties.

\author{
Acknowledgements \\ Not applicable. \\ Funding \\ This work is supported by the Natural Sciences and Engineering Research Council of Canada (GP249507[NS] and \\ RGPIN-2017-05321 [Md]).
}

\title{
Availability of data and materials
}

Not applicable.

\section{Competing interests}

The authors declare that they have no competing interests.

\section{Authors' contributions}

The theory of the Hahn asymptotic iteration method was developed by NS and LM, the confirmation of the underling mathematics was done by MD. examples were discussed and presented by the three authors. All authors read and approved the final manuscript.

\section{Publisher's Note}

Springer Nature remains neutral with regard to jurisdictional claims in published maps and institutional affiliations.

Received: 30 October 2020 Accepted: 19 July 2021 Published online: 30 July 2021

\section{References}

1. Hahn, W.: Über orthogonalpolynome, die q-differenzenlgleichungen genügen. Math. Nachr. 2, 4-34 (1949)

2. Hahn, W.: Ein beitrag zur theorie der orthogonalpolynome. Monatshefte Math. 95, 19-24 (1983)

3. Jackson, F.H.: q-Difference equations. Am. J. Math. 32, 305-314 (1910)

4. Gasper, G., Rahman, M.: Basic Hypergeometric Series, 2nd edn. Cambridge University Press, Cambridge (2004)

5. Annaby, M.H., Mansour, Z.S.: q-Taylor and interpolation series for Jackson q-difference operators. J. Math. Anal. Appl. 344, 472-483 (2008)

6. Andrews, G.E., Askey, R., Roy, R.: Special Functions. Cambridge University Press, Cambridge (1999)

7. Ismail, M.E.H., Stanton, D.: q-Series from a Contemporary Perspective. Contemp. Math., vol. 254. Am. Math. Soc, Providence (2000)

8. Bird, M.T.: On generalizations of sum formulas of the Euler-Maclaurin type. Am. J. Math. 58, 487-503 (1936)

9. Jordan, C.: Calculus of Finite Differences, 3rd edn. Chelsea, New York (1965)

10. Annaby, M.H., Hamza, A.E., Aldwoah, K.A.: Hahn difference operator and associated Jackson-Nörlund integrals. J. Optim. Theory Appl. 154, 133-153 (2012)

11. Aldwoah, K.A.: Generalized time scales and associated difference equations. Ph.D. thesis, Cairo University (2009)

12. Hamza, A.E., Sarhan, A.-S.M., Shehata, E.M., Aldwoah, K.A.: A general quantum difference calculus. Adv. Differ. Equ. 2015, $182(2015)$

13. Álvarez-Nodarse, R.: On characterizations of classical polynomials. J. Comput. Appl. Math. 196, 320-337 (2006)

14. Costas-Santos, R.S., Marcellán, F.: Second structure relation for $q$-semiclassical polynomials of the Hahn tableau. J. Math. Anal. Appl. 329, 206-228 (2007)

15. Dobrogowska, A., Odzijewicz, A.: Second order q-difference equations solvable by factorization method. J. Comput. Appl. Math. 193, 319-346 (2006)

16. Kwon, K.H., Lee, D.W., Park, S.B., Yoo, B.H.: Hahn class orthogonal polynomials. Kyungpook Math. J. 38, $259-281$ (1998)

17. Petronilho, J.: Generic formulas for the values at the singular points of some special monic classical $H_{q, w}$-orthogonal polynomials. J. Comput. Appl. Math. 205, 314-324 (2007)

18. Malinowskaa, A.B., Martins, N.: Generalized transversality conditions for the Hahn quantum variational calculus. Optimization 62, 323-344 (2013)

19. Álvarez-Nodarse, R., Castillo, K., Mbouna, D., Petronilho, J.: On classical orthogonal polynomials related to Hahn's operator. Integral Transforms Spec. Funct. 31, 487-505 (2020). https://doi.org/10.1080/10652469.2019.1702981

20. Ciftci, H., Hall, R.L., Saad, N.: Asymptotic iteration method for eigenvalue problems. J. Phys. A 36, 11807-11816 (2003)

21. Saad, N., Hall, R.L., Ciftci, H.: Criterion for polynomial solutions to a class of linear differential equations of second order. J. Phys. A 39, 13445-13454 (2006)

22. Znojil, M.: A method of constructive quantum mechanics of remarkable hidden beauty. J. Phys. A, Math. Theor. 49, 451003 (2016)

23. Champion, B., Hall, R.L., Saad, N.: Asymptotic iteration method for singular potentials. Int. J. Mod. Phys. A 23, 1405-1415 (2008)

24. Hall, R.L., Saad, N.: Schrödinger spectrum generated by the Cornell potential. Open Phys. 13, 83-89 (2015)

25. Ismail, M.E.H., Saad, N.: The asymptotic iteration method revisited. J. Math. Phys. 61, 033501 (2020). https://doi.org/10.1063/1.5117143

26. Ismail, M.E.H., Saad, N.: A discrete and q asymptotic iteration method. J. Differ. Equ. Appl. 26(4), 488-509 (2020). https://doi.org/10.1080/10236198.2020.1748021

27. Ismail, M.E.H.: Classical and Quantum Orthogonal Polynomials in One Variable. Cambridge University Press, Cambridge (2005) 
28. Koekoek, R., Lesky, O.A., Swarttouw, R.F.: Hypergeometric Orthogonal Polynomials and Their q-Analogues. Springer Monographs in Mathematics. Springer, Berlin (2010)

29. Brikshavana, T., Sitthiwirattham, T.: On fractional Hahn calculus. Adv. Differ. Equ. 2017, 354 (2017)

30. Patanarapeelert, N., Sitthiwirattham, T.: On nonlocal Robin boundary value problems for Riemann-Liouville fractional Hahn integrodifference equation. Bound. Value Probl. 2018, 46 (2018)

31. Sriphanomwan, U., Tariboon, J., Patanarapeeler, N., Ntouyas, S.K., Sitthiwirattham, T.: Nonlocal boundary value problems for second order nonlinear Hahn integro-difference equations with Hahn integral boundary conditions. Adv. Differ. Equ. 2017, 170 (2017)

32. Sitthiwirattham, T:: Nonlocal three-point boundary value problems for nonlinear second-order Hahn difference equations with two different q,w-derivatives. Adv. Differ. Equ. 2016, 116 (2016)

33. Gasper, G., Rahman, M.: Basic Hypergeometric Series. Cambridge University Press, Cambridge (2004)

34. Oraby, K., Hamza, A.: Taylor theory associated with Hahn difference operator. J. Inequal. Appl. 2020, 124 (2020)

35. Hamza, A.E., Ahmed, S.M.: Existence and uniqueness of solutions of Hahn difference equations. Adv. Differ. Equ. 2013, 316 (2013). https://doi.org/10.1186/1687-1847-2013-316

36. Hamza, A.E., Abdelkhaliq, M.M.: Hahn difference equations in Banach algebras. Adv. Differ. Equ. 2016, 161 (2016), https://doi.org/10.1186/s13662-016-0886-3

37. Tariboon, J., Ntouyas, S.K., Sudsutad, W.: New concepts of Hahn calculus and impulsive Hahn difference equations. Adv. Differ. Equ. 2016, 255 (2016). https://doi.org/10.1186/s13662-016-0982-4

38. Allahverdiev, B.P., Tuna, H.: Spectral theory of singular Hahn difference equation of the Sturm-Liouville type. Commun. Math. 28, 13-25 (2020). https://doi.org/10.2478/cm-2020-0002

39. Hamza, A.E., Ahmed, S.M.: Theory of linear Hahn difference equations. J. Adv. Math. 4, 441-461 (2013)

40. Abu-Risha, M.H., Annaby, M.H., Ismail, M.E.H., Mansour, Z.S.: Linear q-difference equations. Z. Anal. Anwend. 26 , 481-494 (2007)

\section{Submit your manuscript to a SpringerOpen ${ }^{\circ}$ journal and benefit from:}

- Convenient online submission

- Rigorous peer review

- Open access: articles freely available online

- High visibility within the field

- Retaining the copyright to your article

Submit your next manuscript at $\boldsymbol{s p r i n g e r o p e n . c o m ~}$ 